\title{
On algebraic dependence of cosmological parameters
}

\author{
Zarko Mijajlovic $^{1}$ and Danijela Brankovic ${ }^{2}$ \\ ${ }^{1}$ University of Belgrade Faculty of Mathematics \\ ${ }^{2}$ University of Belgrade School of Electrical Engineering
}

April 27, 2020

\begin{abstract}
We show that the inverse function of the scale factor $\mathrm{a}(\mathrm{t})$ can be represented as an elliptic integral with a parameter. Using algebraic dependencies between cosmological parameters and the obtained inverse function formula, we compute in a uniform way some special events in the universe's evolution.
\end{abstract}

Hosted file On algebraic dependence of cosmological parameters.pdf available at https://authorea.com/users/315216/articles/ on-algebraic-dependence-of-cosmological-parameters

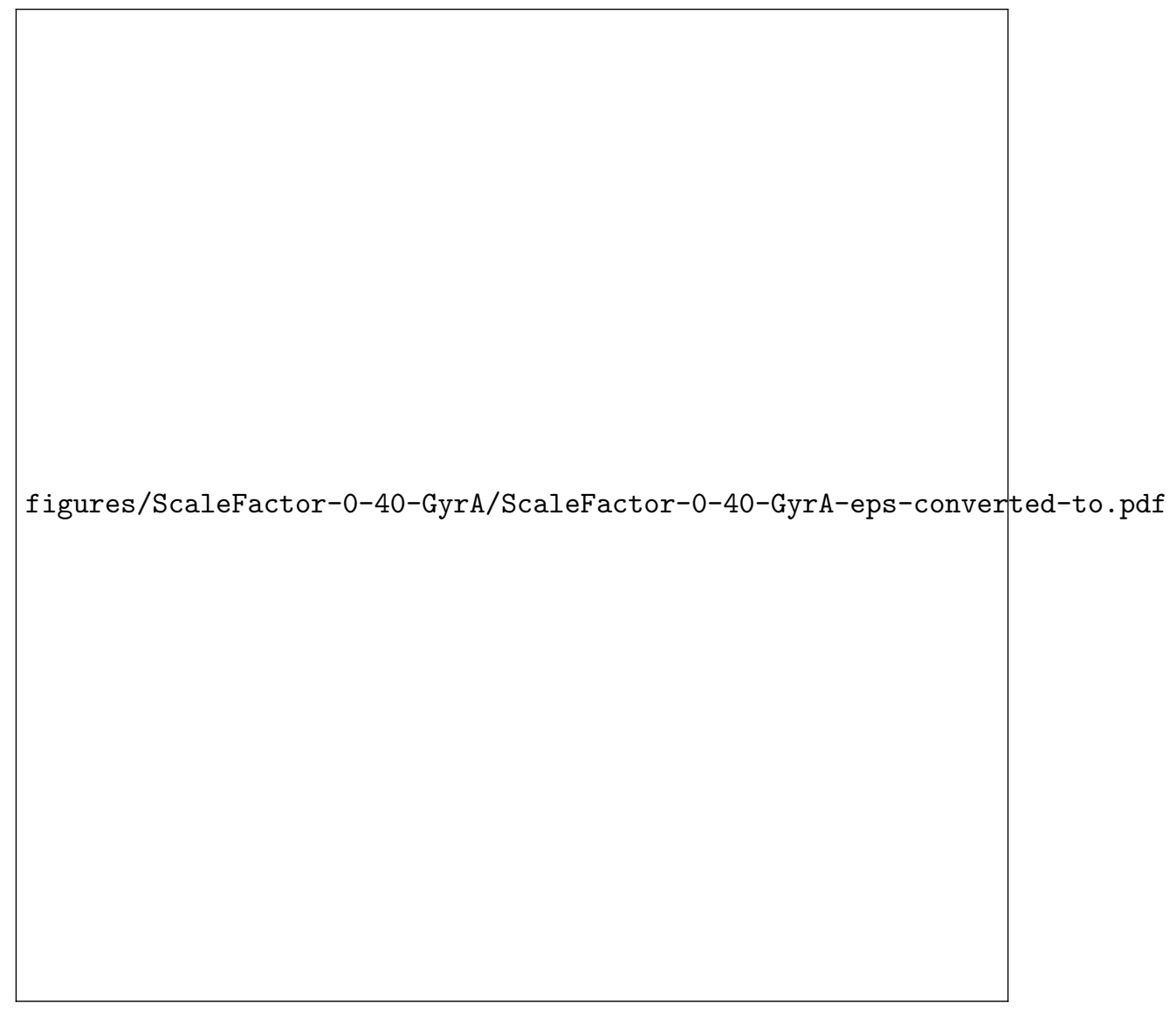

